\title{
Two unconditional stable schemes for simulation of heat equation on manifold using DEC
}

\author{
Zheng $\mathrm{Xie}^{1 *}$ Yujie $\mathrm{Ma}^{2 \dagger}$ \\ 1. Center of Mathematical Sciences Zhejiang University (310027), China \\ 2. Key Laboratory of Mathematics Mechanization, \\ Chinese Academy of Sciences, (100090), China
}

\begin{abstract}
To predict the heat diffusion in a given region over time, it is often necessary to find the numerical solution for heat equation. With the techniques of discrete differential calculus, we propose two unconditional stable numerical schemes for simulation heat equation on space manifold and time. The analysis of their stability and error is accomplished by the use of maximum principle.
\end{abstract}

Keywords: Discrete exterior calculus, Discrete manifold, Heat equation.

PACS(2010): 44.05.+e, 02.30.Jr, 02.30.Mv, 02.40.Vh

\section{Introduction}

To investigate the predictions of heat equation it is often necessary to approximate its solution numerically [1,2]. Discrete exterior calculus (DEC) constitutes a discrete realization of the exterior differential forms structure, and therefore, the right framework in which to develop a discretization of exterior differential system [3 10. The operators in this system such as Hodge star, exterior derivative, and Laplace operator can also be naturally discretized by DEC. The techniques of DEC can be used to construct a conditional stable scheme for heat equation [11. The stable condition is a very severe restriction, and implies that very many time steps will be necessary to follow the solution over a reasonably large time interval.

\footnotetext{
${ }^{*}$ E-mail: lenozhengxie@yahoo.com.cn

${ }^{\dagger}$ E-mail: yjma@mmrc.iss.ac.cn This work is partially supported by CPSFFP (No. 20090460102) NKBRPC (No. 2004CB318000) and NNSFC (No. 10871170)
} 
In this paper we shall propose two unconditional stable schemes for the numerical solution of heat equation in space manifold and the time, namely implicit and semi-implicit DEC schemes. The analysis of their error is accomplished by the use of maximum principle. The methods proposed here can be extended to problems with general boundary condition as explicit scheme, then to general linear and nonlinear parabolic equations.

\section{Implicit DEC scheme for heat equation}

For a function $\psi(x, y, z, t)$ of three spatial variables $(x, y, z)$ and the time variable $t$, the heat equation is

$$
\rho c \frac{\partial \psi}{\partial t}-k\left(\frac{\partial^{2} \psi}{\partial x^{2}}+\frac{\partial^{2} \psi}{\partial y^{2}}+\frac{\partial^{2} \psi}{\partial z^{2}}\right)-\rho Q=0
$$

with boundary conditions where $\psi$ is the temperature, $\rho$ is the material density, $c$ is the material specific heat, $k$ is the thermal conductivity, $Q$ is the internal heat source density.

The DEC method can approximate Laplace operator as

$$
\Delta \approx *^{-1} d^{T} *+d^{T} * d,
$$

where $d$ is the matrix of discrete exterior derivative, $*$ is the matrix of discrete Hodge star operator. The explicit DEC scheme for heat equation uses the forward time difference for the temporal derivative of Eq.(1), which is conditional stable [11]. If we need to refine the space mesh to improve the accuracy of the solution the amount of work involved increases very rapidly, since we shall also reduce the length of time step. Now, we show how the use of a backward time difference gives a difference scheme which avoids this restriction.

Given 2D or 3D space manifold with a smooth boundary, a simplicial mesh is a tessellation of it by triangular or tetrahedra, satisfies the condition that any two of them may intersect along a common face, edge or vertice. For some situations, a source having azimuthal symmetry about its axis is considered. In this case, we only need to consider 2D triangular discrete manifold as the space. The 3D case can also be done in the same way.

At first, we define some values on mesh. Take Fig.1 as an example for 2D mesh, in which $0, A, \ldots, F$ are vertices, $1,2, \ldots, 6$ are the circumcenters of triangles, $a, b, \ldots, f$ are the circumcenters of edges. Denote $l_{i j}$ as the length 
of line segment $(i, j)$ and $A_{i j k}$ as the area of triangle $(i, j, k)$.

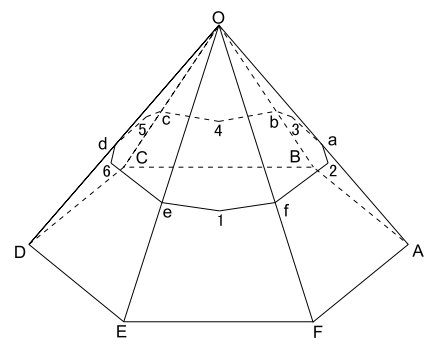

Figure.1

Define

$$
l_{12}:=l_{1 f}+l_{2 f}, l_{23}:=l_{2 a}+l_{3 a}, \ldots, l_{61}:=l_{6 e}+l_{1 e},
$$

and

$$
P_{123456}:=A_{01 f}+A_{02 f}+\cdots+A_{01 e} .
$$

The Laplace operator acting on $\psi$ at vertice 0 approximated by DEC is

$$
\begin{aligned}
\Delta \psi_{0} \approx & \frac{1}{P_{123456}}\left(\frac{l_{23}}{l_{A 0}}\left(\psi_{A}-\psi_{0}\right)+\frac{l_{34}}{l_{B 0}}\left(\psi_{B}-\psi_{0}\right)+\frac{l_{45}}{l_{C 0}}\left(\psi_{C}-\psi_{0}\right)\right. \\
& \left.+\frac{l_{56}}{l_{D 0}}\left(\psi_{D}-\psi_{0}\right)+\frac{l_{16}}{l_{E 0}}\left(\psi_{E}-\psi_{0}\right)+\frac{l_{12}}{l_{F 0}}\left(\psi_{F}-\psi_{0}\right)\right) .
\end{aligned}
$$

If we use the backward time difference, we obtain the implicit DEC scheme for Eq.(1) as follows:

$$
\begin{aligned}
\psi_{0}^{n+1}= & \psi_{0}^{n}+\frac{k \Delta t}{c \rho P_{123456}}\left(\frac{l_{23}}{l_{A 0}}\left(\psi_{A}^{n+1}-\psi_{0}^{n+1}\right)+\frac{l_{34}}{l_{B 0}}\left(\psi_{B}^{n+1}-\psi_{0}^{n+1}\right)\right. \\
& +\frac{l_{45}}{l_{C 0}}\left(\psi_{C}^{n+1}-\psi_{0}^{n+1}\right)+\frac{l_{56}}{l_{D 0}}\left(\psi_{D}^{n+1}-\psi_{0}^{n+1}\right)+\frac{l_{16}}{l_{E 0}}\left(\psi_{E}^{n+1}-\psi_{0}^{n+1}\right) \\
& \left.+\frac{l_{12}}{l_{F 0}}\left(\psi_{F}^{n+1}-\psi_{0}^{n+1}\right)\right)+\frac{\Delta t}{c} \rho Q_{0}^{n} .
\end{aligned}
$$

Now, we use maximum principle to prove the unconditional stability for scheme (2). Given a perturbation $\varepsilon_{i}^{n}$ on each $\psi_{i}^{n}$, the relation between $\varepsilon_{i}^{n}$ and $\varepsilon_{i}^{n+1}$ can be induced from scheme (2) as follows:

$$
\begin{aligned}
\varepsilon_{0}^{n+1}= & \varepsilon_{0}^{n}-\frac{\Delta t}{P_{123456}}\left(\frac{l_{23}}{l_{A 0}}+\frac{l_{34}}{l_{B 0}}+\frac{l_{45}}{l_{C 0}}+\frac{l_{56}}{l_{D 0}}+\frac{l_{16}}{l_{E 0}}+\frac{l_{12}}{l_{F 0}}\right) \varepsilon_{0}^{n+1} \\
& +\frac{\Delta t}{P_{123456}}\left(\frac{l_{23}}{l_{A 0}} \varepsilon_{A}^{n+1}+\frac{l_{34}}{l_{B 0}} \varepsilon_{B}^{n+1}+\frac{l_{45}}{l_{C 0}} \varepsilon_{C}^{n+1}+\frac{l_{56}}{l_{D 0}} \varepsilon_{D}^{n+1}\right. \\
& \left.+\frac{l_{16}}{l_{E 0}} \varepsilon_{E}^{n+1}+\frac{l_{12}}{l_{F 0}} \varepsilon_{F}^{n+1}\right) .
\end{aligned}
$$


If we introduce the maximum perturbation at $n$ time step by writing

$$
\left|\varepsilon^{n}\right|=\operatorname{Max}_{i \in V}\left|\varepsilon_{i}^{n}\right|
$$

we can obtain

$$
\left|\varepsilon_{0}^{n+1}\right| \leq \frac{\left|\varepsilon^{n}\right|+\frac{\Delta t}{P_{123456}}\left(\frac{l_{23}}{l_{A 0}}+\frac{l_{34}}{l_{B 0}}+\frac{l_{45}}{l_{C 0}}+\frac{l_{56}}{l_{D 0}}+\frac{l_{16}}{l_{E 0}}+\frac{l_{12}}{l_{F 0}}\right)\left|\varepsilon^{n+1}\right|}{1+\frac{\Delta t}{P_{123456}}\left(\frac{l_{23}}{l_{A 0}}+\frac{l_{34}}{l_{B 0}}+\frac{l_{45}}{l_{C 0}}+\frac{l_{56}}{l_{D 0}}+\frac{l_{16}}{l_{E 0}}+\frac{l_{12}}{l_{F 0}}\right)} .
$$

Inequality (3) holds for all vertices in grid, therefore

$$
\left|\varepsilon^{n+1}\right| \leq\left|\varepsilon^{n}\right| .
$$

That is to say scheme (2) is unconditional stability. Longer time step can be used than in explicit DEC scheme.

By the definition of truncation error, the solution $\tilde{\psi}$ of Eq.(1) satisfies the same relation as scheme (2) except for an additional term $O\left((\Delta t)^{2}+l^{2}\right)$ on the right hand side. Thus the error $X_{i}^{n}=\tilde{\psi}_{i}^{n}-\psi_{i}^{n}$ is determined from the relation

$$
\begin{aligned}
X_{0}^{n+1}= & X_{0}^{n}-\frac{\Delta t}{P_{123456}}\left(\frac{l_{23}}{l_{A 0}}+\frac{l_{34}}{l_{B 0}}+\frac{l_{45}}{l_{C 0}}+\frac{l_{56}}{l_{D 0}}+\frac{l_{16}}{l_{E 0}}+\frac{l_{12}}{l_{F 0}}\right) X_{0}^{n+1} \\
& +\frac{\Delta t}{P_{123456}}\left(\frac{l_{23}}{l_{A 0}} X_{A}^{n+1}+\frac{l_{34}}{l_{B 0}} X_{B}^{n+1}+\frac{l_{45}}{l_{C 0}} X_{C}^{n+1}+\frac{l_{56}}{l_{D 0}} X_{D}^{n+1}\right. \\
& \left.+\frac{l_{16}}{l_{E 0}} X_{E}^{n+1}+\frac{l_{12}}{l_{F 0}} X_{F}^{n+1}\right)+O\left((\Delta t)^{2}+l^{2}\right)
\end{aligned}
$$

Define

$$
\left|X^{n}\right|=\operatorname{Max}_{i \in V}\left|X_{i}^{n}\right|
$$

It follows that

$$
\left|X_{0}^{n+1}\right| \leq\left|X^{n}\right|+O\left((\Delta t)^{2}+l^{2}\right)
$$

and hence that

$$
\left|X^{n+1}\right| \leq\left|X^{n}\right|+O\left((\Delta t)^{2}+l^{2}\right),
$$

so that, since $X^{0}=0$,

$$
\left|X^{n+1}\right| \leq O\left((\Delta t)^{2}+l^{2}\right),
$$

and this tends to 0 along the refinement path under the assumed hypotheses. So far we have assumed that numerical errors arise from the truncation errors of the finite difference approximations, but that the boundary values are used exactly. 


\section{Semi-implicit DEC scheme for heat equation}

Implicit DEC scheme is not so easy to use as the explicit DEC scheme. We must solve a system of equations to give the values simultaneously, which will cost a lot of time. Now we propose a semi-implicit scheme, which combines the virtues of explicit scheme (direct calculation) and implicit scheme (unconditional stability). This scheme is that the calculated element using next point of time whereas the other elements using current point of time. Hence the semi-implicit scheme of Eq.(1) is

$$
\begin{aligned}
\psi_{0}^{n+1}= & \psi_{0}^{n}+\frac{k \Delta t}{c \rho\left|P_{123456}\right|}\left(\frac{l_{23}}{l_{A 0}}\left(\psi_{A}^{n}-\psi_{0}^{n+1}\right)+\frac{l_{34}}{l_{B 0}}\left(\psi_{B}^{n}-\psi_{0}^{n+1}\right)\right. \\
& +\frac{l_{45}}{l_{C 0}}\left(\psi_{C}^{n}-\psi_{0}^{n+1}\right)+\frac{l_{56}}{l_{D 0}}\left(\psi_{D}^{n}-\psi_{0}^{n+1}\right)+\frac{l_{16}}{l_{E 0}}\left(\psi_{E}^{n}-\psi_{0}^{n+1}\right) \\
& \left.+\frac{l_{12}}{l_{F 0}}\left(\psi_{F}^{n}-\psi_{0}^{n+1}\right)\right)+\frac{\Delta t}{c} \rho Q_{0}^{n} .
\end{aligned}
$$

It can be rewritten as

$$
\psi_{0}^{n+1}=\frac{\psi_{0}^{n}+\frac{k \Delta t}{c \rho P_{123456}}\left(\frac{l_{23}}{l_{A 0}} \psi_{A}+\frac{l_{34}}{l_{B 0}} \psi_{B}^{n}+\frac{l_{45}}{l_{C 0}} \psi_{C}^{n}+\frac{l_{56}}{l_{D 0}} \psi_{D}^{n}+\frac{l_{16}}{l_{E 0}} \psi_{E}^{n}+\frac{l_{12}}{l_{F 0}} \psi_{F}^{n}\right)+\frac{\Delta t}{c} \rho Q_{0}^{n}}{1+\frac{\Delta t}{P_{123456}}\left(\frac{l_{23}}{l_{A 0}}+\frac{l_{34}}{l_{B 0}}+\frac{l_{45}}{l_{C 0}}+\frac{l_{56}}{l_{D 0}}+\frac{l_{16}}{l_{E 0}}+\frac{l_{12}}{l_{F 0}}\right)} .
$$

Now, we consider the stability and convergence for scheme (4). The relation between perturbation $\varepsilon_{i}^{n}$ and $\varepsilon_{i}^{n+1}$ can be induced from scheme (4) as follows:

$$
\varepsilon_{0}^{n+1}=\frac{\varepsilon_{0}^{n}+\frac{k \Delta t}{c \rho P_{123456}}\left(\frac{l_{23}}{l_{A 0}} \varepsilon_{A}^{n}+\frac{l_{34}}{l_{B 0}} \varepsilon_{B}^{n}+\frac{l_{45}}{l_{C 0}} \varepsilon_{C}^{n}+\frac{l_{56}}{l_{D 0}} \varepsilon_{D}^{n}+\frac{l_{16}}{l_{E 0}} \varepsilon_{E}^{n}+\frac{l_{12}}{l_{F 0}} \varepsilon_{F}^{n}\right)}{1+\frac{k \Delta t}{c \rho P_{123456}}\left(\frac{l_{23}}{l_{A 0}}+\frac{l_{34}}{l_{B 0}}+\frac{l_{45}}{l_{C 0}}+\frac{l_{56}}{l_{D 0}}+\frac{l_{16}}{l_{E 0}}+\frac{l_{12}}{l_{F 0}}\right)} .
$$

Since the coefficients are positive, we can omit the modulus signs in the Eq.(5) to give inequality

$$
\begin{aligned}
\left|\varepsilon_{0}^{n+1}\right| & \leq \frac{1+\frac{k \Delta t}{c \rho P_{123456}}\left(\frac{l_{23}}{l_{A 0}}+\frac{l_{34}}{l_{B 0}}+\frac{l_{45}}{l_{C 0}}+\frac{l_{56}}{l_{D 0}}+\frac{l_{16}}{l_{E 0}}+\frac{l_{12}}{l_{F 0}}\right)}{1+\frac{k \Delta t}{c \rho P_{123456}}\left(\frac{l_{23}}{l_{A 0}}+\frac{l_{34}}{l_{B 0}}+\frac{l_{45}}{l_{C 0}}+\frac{l_{56}}{l_{D 0}}+\frac{l_{16}}{l_{E 0}}+\frac{l_{12}}{l_{F 0}}\right)}\left|\varepsilon^{n}\right| \\
& \leq\left|\varepsilon^{n}\right|
\end{aligned}
$$

Inequality (6) holds for all vertices in mesh, therefore

$$
\left|\varepsilon^{n+1}\right| \leq\left|\varepsilon^{n}\right| .
$$


That is to say scheme(5) is unconditional stability.

The relation of error $X_{i}^{n}$ can derived form scheme (4) as follows:

$$
\begin{aligned}
X_{0}^{n+1}= & \frac{X_{0}^{n}+\frac{k \Delta t}{c \rho P_{123456}}\left(\frac{l_{23}}{l_{A 0}} X_{A}^{n}+\frac{l_{34}}{l_{B 0}} X_{B}^{n}+\frac{l_{45}}{l_{C 0}} X_{C}^{n}+\frac{l_{56}}{l_{D 0}} X_{D}^{n}+\frac{l_{16}}{l_{E 0}} X_{E}^{n}+\frac{l_{12}}{l_{F 0}} X_{F}^{n}\right)}{1+\frac{k \Delta t}{c \rho P_{123456}}\left(\frac{l_{23}}{l_{A 0}}+\frac{l_{34}}{l_{B 0}}+\frac{l_{45}}{l_{C 0}}+\frac{l_{56}}{l_{D 0}}+\frac{l_{16}}{l_{E 0}}+\frac{l_{12}}{l_{F 0}}\right)} \\
& +O\left((\Delta t)^{2}+l^{2}\right) .
\end{aligned}
$$

Because of the nonnegative coefficients, it follows that

$$
\left|X_{0}^{n+1}\right| \leq\left|X^{n}\right|+O\left((\Delta t)^{2}+l^{2}\right)
$$

and hence that

$$
\left|X^{n+1}\right| \leq\left|X^{n}\right|+O\left((\Delta t)^{2}+l^{2}\right),
$$

so that, since $X^{0}=0$,

$$
\left|X^{n+1}\right| \leq O\left((\Delta t)^{2}+l^{2}\right),
$$

and this tends to 0 along the refinement path under the assumed hypotheses. That is to say scheme (5) is convergence.

In scheme (2) and (4), the derivative is approximated by first order difference. Equivalently, $\psi$ is approximated by linear interpolation functions. Consulting the definition about accuracy of finite volume method, we can also say that scheme (2) and (4) have first order temporal and spacial accuracy.

\section{Implementation of semi-implicit DEC scheme}

The implementation of semi-implicit scheme (4) for heat equation is the same as the explicit scheme consisting of the following steps:

1. Set the simulation parameters. These are the dimensions of the computational mesh and the size of the time step, etc.;

2. Initialize the mesh indexes.

3. Assign source.

4. Assign boundary conditions.

5. Compute the value of all spatial nodes and temporarily store the result in the circular buffer for further computation.

6. Visualize the currently computed grid of spatial nodes. 
7. Repeat the process from the step 3, until reach the desired total number of iterations.

In the following examples, the parameters is $\rho=k=c=1$ and the heat source is $\sqrt{t / 500}$. The examples in Fig.2 and Fig.3 show that the diffusion of heat on surfaces of dragon and torus. The temperature $\psi>1$ in red domain, $0<\psi \leq 1$ in yellow domain, and $\psi=0$ in blue domain.
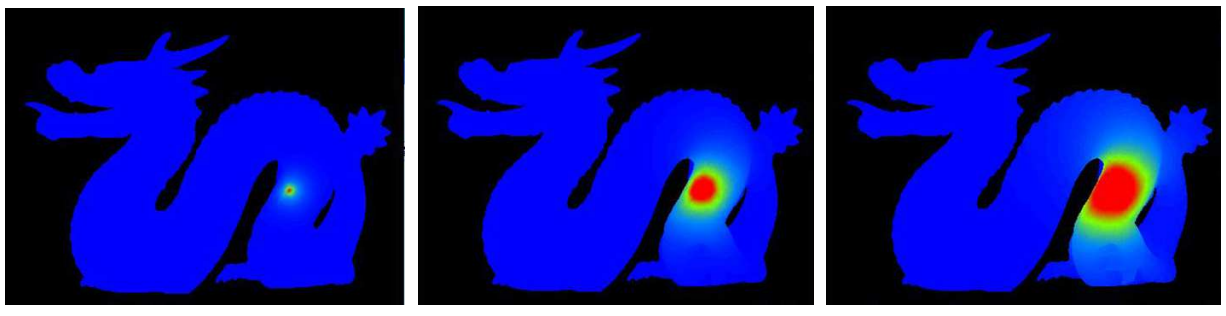

Figure.2
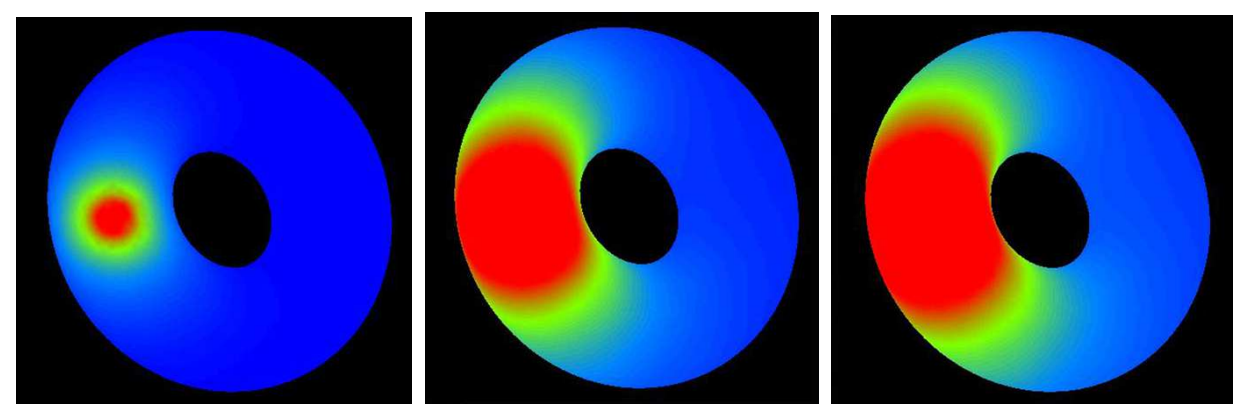

Figure.3

\section{References}

[1] K.W. Morton, D.F. Mayers, Numerical Solution of Partial Differential Equations: An Introduction 2nd Edition, Cambridge University Press, (2005).

[2] S. Larsson, V. Thomée, Parial differential equations with numercial methods, Springer, (2009).

[3] H. Whitney, Geometric integration theory. Princeton University Press, Princeton, (1957).

[4] A. Bossavit, L. Kettunen, : Yee-like schemes on a tetrahedral mesh, with diagonal lumping. Int. J. Numer. Modell. 12(1-2), 129142 (1999). 
[5] D.N. Arnold, R.S. Falk, R. Winther, Finite element exterior calculus, homological techniques, and applications. Acta Numer. 15, 1-155 (2006).

[6] M. Meyer, M. Desbrun, P. Schröder, A.H. Barr, Discrete differential geometry operators for triangulated 2-manifolds. In InternationalWorkshop on Visualization and Mathematics, VisMath, (2002).

[7] M. Desbrun, A.N. Hirani, M. Leok, J. E. Marsden, Discrete exterior calculus arXiv: math.DG/0508341.

[8] J. M. Hyman, M. Shashkov, Natural discretizations for the divergence, gradient, and curl on logically rectangular grids. Comput. Math. Appl., 33(4):81-104, (1997).

[9] R. Hiptmair, Discrete Hodge operators, Numer. Math., 90(2):265-289, (2001).

[10] M. Leok, Foundations of computational geometric mechanics. Ph.D. thesis, California Institute of Technology (2004). URL http://resolver.caltech.edu/ CaltechETD:etd-03022004-000251.

[11] Z. Xie, Y.J. Ma, Simulation of Wave Equation on Manifold using DEC, arXiv:0909.5008. 